\title{
Evaluation of a Practical Visual MRI Rating Scale of Brain White Matter Hyperintensities for Clinicians Based on Largest Lesion Size Regardless of Location
}

\author{
K.S. King, R.M. Peshock, M.W. Warren, L. Alhilali, K. Hulsey, R. McColl, M.F. Weiner, C. Ayers, and A. Whittemore
}

O- $=$

\begin{abstract}
BACKGROUND AND PURPOSE: Age-related white matter hyperintensities have prognostic implications, but no accepted clinical standard exists for their assessment. We propose a simple objective visual rating system by using 3 T brain MR imaging.

MATERIALS AND METHODS: MR imaging from 559 participants was processed by using an automated method to determine WMH volumes and evaluated with a new visual rating scale based on the single largest WMH lesion diameter regardless of location. The reproducibility of the visual system was assessed. The association of WMH visual scores and automated volumes was then compared with cognitive scores from the Montreal Cognitive Assessment, which was available for 510 participants.

RESULTS: Inter-reader reproducibility was good for subsamples with both high $(n=52)$ and low $(n=40)$ prevalence of large automated WMH volumes (agreement of $67 \%$ and $87.5 \%, \kappa=0.71$ and 0.76 , respectively). Correlation between increased WMH and cognitive deficit measurements was equal for our visual ratings and automated volumes (Spearman $\rho=0.118$ and $0.109 ; P$ values $=0.008$ and 0.014 , respectively). The visual scale retained a significant association with MoCA score after adjusting for age, sex, and education (standardized $\beta=-0.087, P=.042)$.
\end{abstract}

CONCLUSIONS: We propose a simple visual WMH scoring system suitable for use as a baseline evaluation in clinical practice.

ABBREVIATIONS: MoCA = Montreal Cognitive Assessment; $\mathrm{WMH}=$ white matter hyperintensity

B rain WMHs are increasingly detected due to greater imaging utilization, the aging of our population, and the higher sensitivity of 3T MR imaging. ${ }^{1}$ Objective determination of disease severity is increasingly important, as mounting evidence implicates $\mathrm{WMH}$ severity as a risk factor for motor and cognitive decline, dementia, stroke, and death. ${ }^{2-7}$ We have developed a simple rat-

Received June 5, 2012; accepted after revision June 24.

From the Department of Radiology (K.S.K., A.W., R.M.P., K.H., R.M.), Department of Internal Medicine (R.M.P.), and Reynolds Cardiovascular Clinical Research Center (C.A., R.M.P.), University of Texas Southwestern Medical Center, Dallas, Texas; Department of Radiology (L.A.), University of Pittsburgh Medical Center, Pittsburgh, Pennsylvania; and Departments of Psychiatry and Neurology (M.F.W., M.W.W.), University of Texas Southwestern Medical Center at Dallas.

This paper was supported in part by grants KL2TR000453 and ULITR000451 from the National Center for Advancing Translational Sciences, National Institutes of Health.

Please address correspondence to Kevin S. King, UT Southwestern Department of Radiology, 5323 Harry Hines Blvd, Dallas, TX 75390; e-mail: Kevin.King@ utsouthwestern.edu

- Indicates open access to non-subscribers at www.ajnr.org

$\equiv$ Indicates article with supplemental on-line tables.

Indicates article with supplemental on-line figures.

http://dx.doi.org/10.3174/ajnr.A3283 ing system with the aim of reducing the considerable variability in white matter hyperintensity assessment in clinical practice. ${ }^{8}$

We adapted our system from the objective grading criteria for deep WMH developed by Gouw et al. Their system has a significant association with cognitive and physical impairment, equivalent to a complex visual scale with WMH localization and WMH volume quantification. ${ }^{9}$ Their use of simple size measurements for grading is desirable as it obviates the need for standard reference images or expert instruction and reduces subjectivity. Their scale is limited, however, in that it does not incorporate rating of periventricular WMH. This is problematic as several large studies evaluating cognitive outcomes have a significant association with periventricular but not deep $\mathrm{WMH},{ }^{3,10-12}$ though this may be due to periventricular WMH more closely correlating with total white matter burden. ${ }^{13}$

Periventricular WMH was initially thought to have a completely distinct etiology from deep WMH. ${ }^{14}$ Later, Fazekas et al ${ }^{15}$ found that advanced (defined as "irregular") periventricular $\mathrm{WMH}$ demonstrated similar ischemic changes at pathology as seen in advanced deep WMH indicating equivalent disease severity. Subsequent studies have also demonstrated that periventricular WMHs extending farther from the ventricular surface are as- 
sociated with lipohyalinosis and ischemic changes whereas thin bands of periventricular WMH $<3 \mathrm{~mm}$ in thickness are likely a normal finding or associated with alterations in the subependymal lining. ${ }^{1,13,15}$

De Carli et al $^{13}$ evaluated visual methods of WMH grading and found lesion classification as periventricular or deep, based on axial images, to be inaccurate. Lesions characterized as deep were often seen to abut the ventricle when examined in multiple planes. Spatial analysis of segmented WMH failed to identify distinct populations of deep versus periventricular disease. Periventricular and deep WMH were found to correlate highly with each other and with the overall WMH burden, favoring a common underlying etiology. Thus, the rationale for differentiating periventricular and deep WMH is increasingly unclear, and its implementation is often imprecise. This argues for rating deep and periventricular WMH jointly.

We assessed a modification of the objective grading system developed by Gouw et $\mathrm{al}^{9}$ by applying its simple size criteria to the assessment of WMH in determining a global disease score based on the largest lesion identified without regard to deep or periventricular location. Reproducibility was assessed among groups with relatively high and low median WMH volume by automated segmentation as lesion load may affect test reliability. ${ }^{16}$ We compared the association between our WMH visual scale and automated volumes with scores from the MoCA. ${ }^{17}$

\section{MATERIALS AND METHODS \\ Participants}

A subject sample of 563 individuals was drawn from a subset of the Dallas Heart Study. ${ }^{18}$ Each participant gave written consent to participate in the study under a protocol approved by the Institutional Review Board. Sample size was chosen to be comparable to or greater than that used in similar published work. ${ }^{9,19}$ We excluded from further analysis 3 participants with encephalomalacia and one with probable vascular malformation resulting in 559 participants evaluated for this study by a neuroradiologist. Of these, a second radiologist also jointly reviewed 20 images as a training set and, for reproducibility testing, 52 from a sample enriched for larger WMH volumes (discussed in the WMH analysis methods section) and 40 from a random sample out of the 559 total evaluated. From this sample, 510 were also evaluated by using the MoCA.

\section{MR Protocol}

Two-dimensional axial FLAIR images were acquired on a 3T MR imaging system (Achieva, Philips Medical Systems; Best, the Netherlands). Thirty-two sections were acquired with TR/TE/ $\mathrm{TI}=11,000 \mathrm{~ms} / 130 \mathrm{~ms} / 2800 \mathrm{~ms}$, echo-train length $=44$, sensitivity encoding factor $=2, \mathrm{FOV}=250 \mathrm{~mm} \times 250 \mathrm{~mm}, 4-\mathrm{mm}$ section thickness with a 1-mm gap between sections, and matrix of $240 \times 138$ yielding a voxel size of $4 \mathrm{~mm} \times 0.96 \mathrm{~mm} \times 1.33$ $\mathrm{mm}=5.11 \mathrm{~mm}^{3}$.

Brain imaging with 2D FLAIR and 3D magnetization-prepared rapid acquisition of gradient echo were acquired on a $3 \mathrm{~T}$ MR imaging system (Achieva). Two-dimensional FLAIR parameters consisted of axial sections acquired with TR/TE/TI $=11,000$ $\mathrm{ms} / 130 \mathrm{~ms} / 2800 \mathrm{~ms}$, echo-train length $=44$, sensitivity encoding factor $=2, \mathrm{FOV}=250 \mathrm{~mm} \times 250 \mathrm{~mm}, 4-\mathrm{mm}$ section thickness with a 1-mm gap between sections, and matrix of $240 \times 138$ yielding a voxel size of $4 \mathrm{~mm} \times 0.96 \mathrm{~mm} \times 1.33 \mathrm{~mm}=5.11 \mathrm{~mm}^{3}$. Three-dimensional magnetization-prepared rapid acquisition of gradient echo parameters consisted of axial sections reconstructed at 1.0-mm section thickness; TR, $9.6 \mathrm{~ms}$; TE, $5.8 \mathrm{~ms}$; flip angle, $12^{\circ}$; FOV $260 \mathrm{~mm} \times 260 \mathrm{~mm}$.

\section{WMH Analysis}

Quantification of WMH volume ( $\mathrm{mL}$ ) was performed by using an automated segmentation algorithm we developed, which has been previously described ${ }^{20}$ by using the FMRIB Software Library (FSL; http://www.fmrib.ox.ac.uk/fsl). ${ }^{21,22}$

Visual grading was based on the size of the single largest lesion as shown in On-line Table 2. All measurements were taken from axial FLAIR images. The intensity threshold of WMH was defined by an internal standard as greater than that of cortical gray matter. Periventricular and deep WMH are equivalent in our system, and one overall global score is given reflecting the single largest $\mathrm{WMH}$ lesion regardless of location. The size for periventricular WMH is the point of greatest thickness taken perpendicular to the ventricle (On-line Fig 1). The size for deep WMH is the diameter of the largest lesion.

Two investigators jointly reviewed 20 images for a baseline training set then independently read a test set of studies from 52 participants selected to have a range of WMH volumes based on results of our automated analysis. One reviewer reread the first set of images in a randomized order after 1 week. Review of the initial test set revealed disagreement regarding grading of lesions $<3 \mathrm{~mm}$ in diameter. A clarification was incorporated that lesions $<3 \mathrm{~mm}$ in diameter or thickness would not be counted as WMHs. A second group of 40 participants were then chosen randomly for review. One author reread these studies after several months by using a different computer and different lighting to assess the maximum intrarater variability. The 3-mm threshold was applied to the grading of all 559 images used for subsequent analysis.

\section{Cognitive Assessment}

The MoCA is a brief 30-point screening test of global cognitive function. The published cutoff for cognitive impairment is $\leq 25 .{ }^{17}$ For our study an additional cutoff score of $\leq 19$ points was also evaluated, which was one standard deviation below our observed population mean of 23 points. $^{23}$

\section{Statistics}

Calculations were made by using SAS software, version 9.2 (SAS Institute, Cary, North Carolina). Inter- and intrarater reliability was assessed by using a weighted $\kappa$ coefficient reflecting the ascending order of our visual rating scale with values below 0.40 reflecting poor agreement, 0.40 to 0.75 reflecting fair to good agreement, and values above 0.75 reflecting excellent agreement. ${ }^{24}$ Visual ratings by a neuroradiologist (K.S.K.) for 559 participants were correlated with automated $\mathrm{WMH}$ volumes $(\mathrm{mL})$, age (years), and cognition (total MoCA score) by using the Spearman rank correlation. Automated WMH volumes were also correlated with MoCA score by using Spearman rank correlation. Pearson $\chi^{2}$ and Mann-Whitney $U$ tests were used to assess differ- 
Table 1: Age and WMH volume by automated measurement for given WMH grade, $n=559$

\begin{tabular}{|c|c|c|c|c|c|c|c|c|}
\hline \multirow[b]{2}{*}{ Grade } & \multirow[b]{2}{*}{ Number } & \multirow[b]{2}{*}{ Percent } & \multicolumn{3}{|c|}{ Age } & \multicolumn{3}{|c|}{ WMH (ml) } \\
\hline & & & Mean & SD & Range & Median & Q1-Q3 & Range \\
\hline 0 & 241 & $43.1 \%$ & 46.7 & 8.8 & $25-72$ & 0.65 & $0.43-0.91$ & $0.14-2.20$ \\
\hline 1 & 255 & $45.6 \%$ & 51.4 & 8.9 & 27-71 & 1.01 & $0.71-1.42$ & $0.13-5.51$ \\
\hline 2 & 49 & $8.8 \%$ & 58.6 & 7.9 & $37-72$ & 2.16 & $1.42-3.38$ & $0.55-9.69$ \\
\hline 3 & 14 & $2.5 \%$ & 64.2 & 5 & $57-72$ & 13.97 & $7.15-30.18$ & $4.53-83.18$ \\
\hline
\end{tabular}

Table 2: Association between measures of WMH and cognitive function using MoCA cutoffs for cognitive deficit as originally published and as established in our cohort

\begin{tabular}{|c|c|c|c|c|c|c|}
\hline & $\begin{array}{c}\text { Normal } \\
(\mathrm{MoCA}>25)\end{array}$ & $\begin{array}{c}\text { Deficit } \\
\text { (MoCA } \leq 25)\end{array}$ & $P$ Value & $\begin{array}{c}\text { Normal } \\
(\mathrm{MoCA}>19)\end{array}$ & $\begin{array}{c}\text { Deficit } \\
\text { (MoCA } \leq 19)\end{array}$ & $P$ Value \\
\hline $\mathrm{N}(\%)$ & $210(41.2)$ & $300(58.8)$ & & $441(86.5)$ & 69 (13.5) & \\
\hline \multicolumn{7}{|l|}{ Visual Scale ${ }^{a}$} \\
\hline Absent (\%) & 103 & 117 & 0.050 & 199 & 21 & 0.001 \\
\hline Minimal (\%) & 92 & 143 & & 204 & 31 & \\
\hline Moderate (\%) & 12 & 30 & & 29 & 13 & \\
\hline Advanced (\%) & 3 & 10 & & 9 & 4 & \\
\hline $\begin{array}{l}\text { Automated Volume }{ }^{b}(\mathrm{~mL}), \\
\text { median (range) }\end{array}$ & $0.815(0.14-39.64)$ & $0.95(0.13-83.18)$ & 0.014 & $0.90(0.13-83.18)$ & $1.05(0.14-45.48)$ & 0.091 \\
\hline
\end{tabular}

ences in WMH visual scale and automated volumes (respectively) between normal and cognitively impaired groups by using MoCA cutoffs of $\leq 19$ and $\leq 25$. A multivariate linear regression was performed to measure linear association of visual grade with total MoCA score while controlling for age, sex, and education as covariates.

\section{RESULTS}

Among the 559 participants, the average age was $50.7 \pm 9.7$ (mean $\pm \mathrm{SD}$ ) with a range from 25 to 72 years; $52.5 \%$ were women. The ethnic distribution was $43 \%$ black non-Hispanic, $38.9 \%$ white non-Hispanic, $15.5 \%$ Hispanic, and $2.5 \%$ other. The distribution of WMH in our sample was nonparametric with median WMH volume of $0.90 \mathrm{~mL}$ (1st and 3rd quartile, Q1-Q3, of $0.58-1.29 \mathrm{~mL}$ and range $0.20-83.18 \mathrm{~mL}$ ). Fifty-two participants in an initial test sample (mean age $56.3 \pm 10.9$ years) enriched for larger automated WMH volume had a median $\mathrm{WMH}$ volume of $1.64 \mathrm{~mL}$ (Q1-Q3, 0.77-4.88 mL; range $0.20-45.48 \mathrm{~mL}$ ). Forty participants in a second visual reading test group (mean age $48.6 \pm 10.6)$ were randomly selected and had a lower median WMH volume of $0.93 \mathrm{~mL}$ (Q1-Q3, 0.57-1.24 mL; range 0.29$2.01 \mathrm{~mL})$. Scores for the 559 participants rated by a neuroradiologist with corresponding age and $\mathrm{WMH}$ volume are shown in Table 1 .

For the group enriched to have a higher prevalence of large $\mathrm{WMH}$ volumes, interrater agreement was $67 \%(\kappa 0.71 ; 95 \% \mathrm{CI}$, $0.58-0.83$ ), and intrarater agreement was $83 \%(\kappa 0.80 ; 95 \% \mathrm{CI}$, $0.70-0.90)$. For the group with lower prevalence of large WMH volumes, interrater agreement was $88 \%(\kappa 0.78$; 95\% CI, $0.59-$ 0.96 ), and intrarater agreement was 0.81 ( $\kappa 0.67$; $95 \%$ CI, $0.47-$ $0.87)$. Visual WMH grades showed significant correlation with automated volumes (Spearman $\rho=0.57, P<.0001$ ) and age (Spearman $\rho=0.40, P<.0001$ ). Age thresholds for advanced WMH were evident with grade 2 only seen at age 37 and above and grade 3 at age 57 and above. Conversely, absence of significant $\mathrm{WMH}$ (grade 0) was observed even among the oldest members of our study.
Correlations between cognition and WMH were comparable across methods of WMH measurement (Spearman $r=0.118$ and 0.109 ; $P$ values $=0.008$ and 0.014 for the visual rating scale and volumetric assessment, respectively). WMH load by visual and volumetric methods were compared between normal and cognitively impaired groups for both MoCA cutoff points in Table 2. The visual scale retained a significant linear association with MoCA score as the dependent variable in a multivariate linear regression controlling for age, sex, and education (standardized $\beta=-0.087, P=.042)$.

\section{DISCUSSION}

Our WMH visual rating system for MR imaging based on the single largest lesion size regardless of location showed good reproducibility and had significant association with cognitive performance, equivalent to that of automated WMH volumes. The association between the visual WMH rating and MoCA scores persisted after controlling for age, sex, and education.

A defining and controversial aspect of our rating system is that it does not distinguish between periventricular and deep WMH. This reflects the work of DeCarli et al, ${ }^{13}$ which argued that, excluding thin periventricular caps and rims, evidence does not support categorizing WMH as deep or periventricular on MR imaging. We direct readers to their article for a comprehensive discussion. In brief, their study did not identify distinct populations with periventricular versus deep WMH. Rather, it showed WMH burden in both locations to be highly correlated with each other and with total WMH burden. Similarly, a prior pathologic study of WMH noted the distinction between deep and periventricular WMH was often "blurred," preventing a clear distinction. ${ }^{25}$ Evidence supports the presence of a vascular zone at risk for ischemia fed by arterioles extending centripetally from the cortex into the deep white matter then on toward the ventricles without appreciable flow centrifugally out from the ventricles. $^{26-28}$ This at-risk vascular zone encompasses both advanced periventricular and deep $\mathrm{WMH}$ as described on MR imaging. ${ }^{13}$ 
DeCarli et $\mathrm{al}^{13}$ also demonstrated the inaccuracy of localizing WMH based solely on axial imaging, raising the possibility that many rating systems that purport to distinguish deep from periventricular WMH may not in fact achieve that result (On-line Fig 2). It is possible that this deficiency could account for 2 metaanalyses that were not able to verify the reproducibility of reported localized effects for deep versus periventricular $\mathrm{WMH}$, though reproducible associations between total WMH and cognitive outcomes were shown. ${ }^{29,30}$

We do not dispute that more complex grading systems with further localization will have utility for specialists and researchers. A comprehensive accounting of effects attributable to white matter disease by lesion location may need to identify which tracts and functional circuits are disrupted. ${ }^{31-33}$ Our system is not meant to preclude more comprehensive analysis, however, and may in fact be useful by identifying which individuals deserve more detailed evaluation. The prospect that $\mathrm{WMH}$ may have localized effects also does not invalidate the utility of assessing global disease burden. WMHs are indicative of diffuse underlying microvascular disease and are associated with frontal hypometabolism and executive dysfunction regardless of their location. ${ }^{34}$

During the initial evaluation of our scale it became apparent that intensity and size thresholds for WMH were also needed to obtain agreement between different raters. On 3T FLAIR images, focal, well-defined regions of WMH were often surrounded by less well-defined and less intense regions of increased signal (Online Fig 3). Diffusion tensor imaging demonstrates a penumbra of decreasing severity of derangement extending out from $\mathrm{WMH}$ lesions. ${ }^{35}$ At higher field strength, more subtle disease becomes apparent on FLAIR imaging, ${ }^{1}$ which may account for the intensity gradient we observed. We chose to include only more intense regions of white matter abnormality on our $3 \mathrm{~T}$ scans to more closely correlate with prior WMH work conducted on lower field strength MR imaging. We set an internal reference that $\mathrm{WMH}$ lesions must be more intense than cortical gray matter, an inversion of the normal relationship. We also found that a 3-mm size threshold was necessary to reliably distinguish punctuate WMH lesions from background variations in white matter signal intensity. With implementation of these changes, our system demonstrated good to excellent reproducibility in assessing participants with either low or high prevalence for large volumes of WMH.

A potential weakness of our system is that a 4-point scale will not represent the WMH burden with the same fidelity as more volumetric scales, thereby limiting the strength of associations with outcomes. We did not see this in our study, but the makeup of our study population may have influenced this result. Our community-based sample did not include those in institutions such as nursing homes, which likely limited the degree of impairment we observed. It is also possible that a more extensive cognitive assessment would yield improved associations with WMH volumes compared with our scale. Our rating scale will also not likely reflect interval changes in $\mathrm{WMH},{ }^{36}$ but these changes can be noted separately by the routine practice of reviewing prior studies alongside the new ones.

The intent of our grading system is to reduce the variability of white matter hyperintensity assessment in clinical readings.
Adoption of a standardized approach to assessing and reporting lesion severity is necessary to translate knowledge gained from WMH research into useful prognostic information. The results of this study indicate that our simple WMH grading system based on the single largest lesion size regardless of location has good reproducibility and a significant association with cognitive performance, equaling that of automated WMH volumes. Our work supports the validity of our scale in the baseline evaluation of WMH on MR imaging of the brain.

\section{CONCLUSIONS}

We propose a practical visual grading system for WMH based on largest lesion size regardless of location, which has good reproducibility and a significant association with cognitive function equivalent to that of automated WMH volumes. Application of this method using simple size criteria will add clarity to the clinical assessment and communication of WMH severity.

Disclosures: Ronald Peshock—RELATED: Grant: Donald W. Reynolds Foundation; Support for Travel to Meetings for the Study or Other Purposes: Donald W. Reynolds Foundation. * Money paid to institution

\section{REFERENCES}

1. Neema M, Guss ZD, Stankiewicz JM, et al. Normal findings on brain fluid-attenuated inversion recovery MR images at 3T. AJNR Am J Neuroradiol 2009;30:911-16

2. Debette S, Markus HS. The clinical importance of white matter hyperintensities on brain magnetic resonance imaging: systematic review and meta-analysis. BMJ 2010;341:c3666

3. Debette S, Bombois S, Bruandet A, et al. Subcortical hyperintensities are associated with cognitive decline in patients with mild cognitive impairment. Stroke 2007;38:2924-30

4. Baezner H, Blahak C, Poggesi A, et al. Association of gait and balance disorders with age-related white matter changes: the LADIS study. Neurology 2008; 70:935-42

5. Blahak C, Baezner H, Pantoni L, et al. Deep frontal and periventricular age related white matter changes but not basal ganglia and infratentorial hyperintensities are associated with falls: cross sectional results from the LADIS study. J Neurol Neurosurg Psychiatry 2009;80:608-13

6. Inzitari D, Simoni M, Pracucci G, et al. Risk of rapid global functional decline in elderly patients with severe cerebral age-related white matter changes: the LADIS study. Arch Intern Med 2007;167: $81-88$

7. Libon DJ, Price CC, Giovannetti T, et al. Linking MRI hyperintensities with patterns of neuropsychological impairment: evidence for a threshold effect. Stroke 2008;39:806-13

8. Yetkin FZ, Haughton VM, Fischer ME, et al. High-signal foci on MR images of the brain: observer variability in their quantification. $A J R$ Am J Roentgenol 1992;159:185-88

9. Gouw AA, Van der Flier WM, van Straaten EC, et al. Simple versus complex assessment of white matter hyperintensities in relation to physical performance and cognition: the LADIS study. J Neurol 2006;253:1189-96

10. Prins ND, van Dijk EJ, den Heijer T, et al. Cerebral small-vessel disease and decline in information processing speed, executive function and memory. Brain 2005;128:2034-41

11. van den Heuvel DM, ten Dam VH, de Craen AJ, et al. Increase in periventricular white matter hyperintensities parallels decline in mental processing speed in a non-demented elderly population. J Neurol Neurosurg Psychiatry 2006;77:149-53

12. van Dijk EJ, Prins ND, Vrooman HA, et al. Progression of cerebral small vessel disease in relation to risk factors and cognitive consequences: Rotterdam Scan study. Stroke 2008;39:2712-19 
13. DeCarli C, Fletcher E, Ramey V, et al. Anatomical mapping of white matter hyperintensities (WMH): exploring the relationships between periventricular WMH, deep WMH, and total WMH burden. Stroke 2005;36:50-55

14. Fazekas F, Chawluk J, Alavi A, et al. MR signal abnormalities at $1.5 \mathrm{~T}$ in Alzheimer's dementia and normal aging. AJR Am J Roentgenol 1987;149:351-56

15. Fazekas F, Kleinert R, Offenbacher H, et al. Pathologic correlates of incidental MRI white matter signal hyperintensities. Neurology 1993;43:1683-89

16. Wardlaw JM, Ferguson KJ, Graham C. White matter hyperintensities and rating scales-observer reliability varies with lesion load. J Neurol 2004;251:584-90

17. Nasreddine ZS, Phillips NA, Bedirian V, et al. The Montreal Cognitive Assessment, MoCA: a brief screening tool for mild cognitive impairment J Am Geriatr Soc 2005;53:695-99

18. Victor RG, Haley RW, Willett DL, et al. The Dallas Heart Study: a population-based probability sample for the multidisciplinary study of ethnic differences in cardiovascular health. Am J Cardiol 2004;93:1473-80

19. Kapeller $P$, Barber R, Vermeulen RJ, et al. Visual rating of age-related white matter changes on magnetic resonance imaging: scale comparison, interrater agreement, and correlations with quantitative measurements. Stroke 2003;34:441-45

20. Hulsey KM, Gupta M, King KS, et al. Automated quantification of white matter disease extent at $3 \mathrm{~T}$ : comparison with volumetric readings. J Magn Reson Imaging. 2012;36:305-11

21. Woolrich MW, Jbabdi S, Patenaude B, et al. Bayesian analysis of neuroimaging data in FSL. Neuroimage 2009;45:S173-86

22. Smith SM, Jenkinson M, Woolrich MW, et al. Advances in functional and structural MR image analysis and implementation as FSL. Neuroimage Suppl 1 2004;23:S208-19

23. Rossetti HC, Lacritz LH, Cullum CM, et al. Normative data for the Montreal Cognitive Assessment (MoCA) in a population-based sample. Neurology 2011;77:1272-75

24. Fleiss J. Measuring normal scale agreement among many raters. Psychological Bulletin 1971;76:378-82
25. Scheltens P, Barkhof F, Leys D, et al. Histopathologic correlates of white matter changes on MRI in Alzheimer's disease and normal aging. Neurology 1995;45:883-88

26. Nelson MD, Jr, Gonzalez-Gomez I, Gilles FH. Dyke Award. The search for human telencephalic ventriculofugal arteries. AJNR Am J Neuroradiol 1991;12:215-22

27. Nonaka H, Akima M, Hatori T, et al. Microvasculature of the human cerebral white matter: arteries of the deep white matter. Neuropathology 2003;23:111-18

28. Moody DM, Bell MA, Challa VR. Features of the cerebral vascular pattern that predict vulnerability to perfusion or oxygenation deficiency: an anatomic study. AJNR Am J Neuroradiol 1990;11: 431-39

29. Gunning-Dixon FM, Raz N. The cognitive correlates of white matter abnormalities in normal aging: a quantitative review. Neuropsychology 2000;14:224-32

30. Oosterman JM, Sergeant JA, Weinstein HC, et al. Timed executive functions and white matter in aging with and without cardiovascular risk factors. Rev Neurosci 2004;15:439-62

31. Tekin S, Cummings JL. Frontal-subcortical neuronal circuits and clinical neuropsychiatry: an update. J Psychosom Res 2002;53: 647-54

32. Salat DH, Tuch DS, van der Kouwe AJ, et al. White matter pathology isolates the hippocampal formation in Alzheimer's disease. Neurobiol Aging 2010;31:244-56

33. Schmahmann JD, Pandya DN, Wang R, et al. Association fiber pathways of the brain: parallel observations from diffusion spectrum imaging and autoradiography. Brain 2007;130:630-53

34. Tullberg M, Fletcher E, DeCarli C, et al. White matter lesions impair frontal lobe function regardless of their location. Neurology 2004; 63:246-53

35. Maillard P, Fletcher E, Harvey D, et al. White matter hyperintensity penumbra. Stroke 2011;42:1917-22

36. van den Heuvel DM, ten Dam VH, de Craen AJ, et al. Measuring longitudinal white matter changes: comparison of a visual rating scale with a volumetric measurement. AJNR Am J Neuroradiol 2006; 27:875-78 